\title{
Was ist Sozialmanagement? Eine Positionsbestimmung anhand einer Sammelrezension aktueller Lehrbücher
}

\section{Vorbemerkung zur Themenwahl}

Die sogenannte Sozialwirtschaft stellt in Deutschland eine Branche dar, die in der jüngeren Vergangenheit nahezu durchgängig Wachstumspotentiale aufweisen konnte. Die volkswirtschaftliche Bedeutung der Sozialwirtschaft ist beachtlich. Fast 1,5 Mio. Beschäftigte (Vollzeitäquivalente) sind bei den Wohlfahrtsverbänden tätig. Mit zusammen rund 1 Mio. Mitarbeitern sind die Caritas und die Diakonie die größten privaten Arbeitgeber in Deutschland (Zimmer et al 2009, S. 130 f.).

Der Terminus Sozialwirtschaft und das sich darin vollziehende Management werden erst seit wenigen Jahren innerhalb der Branche zunehmend akzeptiert. Diese zögerliche Akzeptanz kann einerseits auf das Fehlen einer gemeinsamen Identität als Branche zurückgeführt werden, da Organisationen der Sozialwirtschaft zwar überwiegend dem Nonprofit-Sektor entstammen, hinzu kommen aber auch gewinnorientierte Unternehmen und je nach Definition auch die Sozialverwaltung. Das Akzeptanzproblem kann andererseits aber auch darauf zurückgeführt werden, dass Anbieter von sozialen Dienstleistungen sich historisch und ideologisch bedingt lange Zeit einer Betrachtung unter wirtschaftlichen Kategorien verweigerten und sich selbst kaum als (Wirtschafts-) Branche wahrnahmen. Ein exemplarischer Blick in das einschlägige Schrifttum fördert daher auch eine Vielfalt von Beiträgen zu Tage, die den Import wirtschaftswissenschaftlichen Denkens in den Sozialbereich mindestens kritisch, wenn nicht sogar strickt ablehnend gegenüberstehen (vgl. z. B. Grams 2000; Albert 2006; Prokla 2007). Es wird bemängelt, dass betriebswirtschaftliche Methoden nicht einfach auf soziale Organisationen übertragen werden können (vgl. z. B. Maelicke 2008, S. 923ff.; Brinkmann 2010, S. 9 f.). Vielmehr geht es darum, einen für den Sozialbereich eigenen managerialen Zugang zu konstruieren. Ein solcher müsste dann einen erkennbaren Input aus dem Theoriefundus der Sozialen Arbeit aufweisen, um den Besonderheitsanspruch zu untermauern und auch die überwiegende Verortung von entsprechenden Studiengängen fast ausnahmslos an den Fachbereichen für Soziale Arbeit zu erklären.

Mehr als zwanzig Jahren nach den ersten Buchveröffentlichungen zum Sozialmanagement (vgl. z. B. Müller-Schöll/Priebke 1989; Flösser/Otto 1992; Gehrmann 1993; Schwarz 1994) soll im Folgenden anhand einer Rezension ausgewählter Lehrbücher der aktuelle inhaltliche Entwicklungsstand des Sozialmanagements untersucht werden.

\section{Kriterien und Literaturauswahl}

Um ein hinlänglich aktuelles Bild gewinnen zu können, werden ausschließlich Lehrbücher berücksichtigt, die ab dem Jahr 2010 erschienen sind. Des Weiteren werden nur solche Titel be- 
rücksichtigt, die ein möglichst breites Themenspektrum abdecken. Publikationen, die sich zwar dem Titel nach mit Sozialmanagement oder Management in sozialen Einrichtungen befassen, faktisch aber ausschließlich nur besondere Arten von Einrichtungen (z. B. Kindertagesstätten) oder spezielle Funktionen (z. B. Personalführung) behandeln, werden ausgeklammert.

$\mathrm{Zu}$ jedem Titel wird die Themenbreite dargestellt und eine inhaltliche Würdigung vorgenommen. Danach erfolgt jeweils eine Gesamtbetrachtung im Lichte des oben skizzierten Besonderheitsanspruchs des Managementzugangs. Die Annahme ist, dass, wenn Sozialmanagement etwas essentiell anderes sein soll als Betriebswirtschaft für den Sozialbereich, dann sollte sich dieses nach über zwanzig Jahren auch in einschlägigen Lehrwerken niederschlagen. Entsprechend den beschriebenen Auswahlkriterien werden folgende Titel besprochen:

- Bieker, R./Vomberg, E. (Hrsg.): Management in der Sozialen Arbeit, Kohlhammer, Stuttgart 2012, $222 \mathrm{~S}$.

- Böttcher, W./Merchel, J.: Einführung in das Bildungs- und Sozialmanagement, Barbara Budrich UTB, Opladen \& Farmington Hills, MI 2010, 256 S.

- Schellberg, K.: Betriebswirtschaftslehre für Sozialunternehmen, 5. Aufl., Ziel, Augsburg 2012, $264 S$.

- Wöhrle, A./Beck, R./Grunwald, K./Schwarz, G./Wendt, W.R.: Grundlagen des Managements in der Sozialwirtschaft, Nomos, Baden-Baden 2013, $250 \mathrm{~S}$.

\section{Die Lehrwerke im Vergleich}

\section{Bieker/Vomberg (Hrsg.): Management in der Sozialen Arbeit}

\section{a) Themenbreite und inhaltliche Analyse}

Das von Rudolf Bieker und Edeltraut Vomberg herausgegebene Werk erscheint als Band 7 der Reihe Grundwissen Soziale Arbeit. Es soll in ,zentrale Felder des Sozialmanagements [einführen,] ... das notwendige Orientierungswissen liefern und zu einer reflektierten Haltung gegenüber betriebswirtschaftlichen Prozessen in der Sozialen Arbeit beitragen“ (S. 5). Diese zentralen Felder werden in insgesamt zehn Kapiteln behandelt:

- Soziale Arbeit und Sozialmanagement (Kolhoff, L., 22 S.)

- Soziale Arbeit im Spannungsfeld der Organisation (Loffing, Ch., 17 S.)

- Projektmanagement in der Sozialen Arbeit (Krewer, A.M./Fernández E. O., 18 S.)

- Sozio-Marketing (Christa, H., 19 S.)

- Personalmanagement in der Sozialen Arbeit (Bode, S., 21 S.)

- Diversity Management und Soziale Arbeit (Kreuzer, M./Kulig, T., 18 S.)

- Qualitätsmanagement in der Sozialen Arbeit (Vomberg, E., 23 S.)

- Aspekte der Wirtschaftlichkeitsrechnung in sozialen Einrichtungen (Heister, W., 23 S.)

- Existenzgründung in der Sozialen Arbeit (Vomberg, E., 18 S.)

- Finanzierung Sozialer Arbeit (Bieker, R., 22 S.) 
Jedes Kapitel enthält ein separates Literaturverzeichnis sowie weiterführende Hinweise für eine vertiefende Einarbeitung.

Der einführende Text Soziale Arbeit und Sozialmanagement erweckt die Erwartung, die ggf. gegenseitige Beeinflussung von wirtschaftlichen und 'sozialen' Denktraditionen näher zu beleuchten. Gleichwohl erfolgt direkt auf der ersten Seite (S. 11) der Hinweis, dass Vertreter Sozialer Arbeit sich mit der Bewirtschaftung knapper Ressourcen befassen müssen, um damit die eigene Einrichtung bei der Behauptung gegenüber anderen Einrichtungen - also wettbewerblich - zu unterstützen. Die Grundausrichtung erscheint hier somit schon vorgegeben. Es folgt ein kurzer Überblick über den Gegenstand, die Historie und Methoden Sozialer Arbeit. Weiter wird der Begriff der ,sozialen Dienstleistungsproduktion“ eingeführt. Dass Kunde wie Anbieter gleichermaßen aktiv bei der Dienstleistungsproduktion beteiligt sind (S. 15), stellt jedoch mitnichten eine Besonderheit von sozialen Dienstleistungen dar. Gleiches gilt auch für die Sachzieldominanz, die erstens nur für Nonprofit Organisationen (NPO) der Sozialwirtschaft gilt und zweitens - weil für alle Arten von NPO gültig - keine explizite Besonderheit sozialer Organisationen darstellt. Inwiefern die Dominanz des Sachzieles auch im Zusammenhang mit dem Thema „Existenzgründung“ (S. 180ff.) gelten kann, ist ohnehin fraglich, weil in dem entsprechenden Kapitel zur Existenzsicherung Soziale Arbeit auch als ,unternehmerische und gewinnorientierte Arbeit“ (S. 180) bezeichnet wird. Eine tatsächliche Besonderheit stellen demgegenüber jedoch ,nicht schlüssige Tauschbeziehungen“ (S. 16) dar, wenn Leistungsempfänger und Kostenträger nicht identisch sind.

In den Textbeiträgen zu den Themen Organisation, Projekt- und Personalmanagement, Marketing, Wirtschaftlichkeitsrechnung, Existenzgründung und Finanzierung wird im Kern Einführungswissen zu diesen Themen aus der Betriebswirtschafts- und/oder Managementlehre dargeboten. Der Bezug zum Sozialbereich wird durchgängig dadurch hergestellt, dass die entsprechenden Ausführungen anhand von Beispielen aus sozialen Einrichtungen oder sozialen Arbeitsbereichen verdeutlicht werden. So werden z. B. im Kapitel zur Organisation Themen wie Ein- und Mehrliniensysteme oder Matrixorganisation genauso behandelt, wie das klassische Drei-PhasenModell zum Organisationswandel nach Kurt Lewin. Im Abschnitt zum (Sozio-) Marketing wird explizit vorangestellt, dass es sich dabei ,um eine Übertragung der erwerbswirtschaftlichen Marketinglehre auf den Bereich der sozialen Dienstleistungen [handelt].“ (S. 71), bevor Themen wie Markt- und SWOT-Analyse oder der Marketing-Mix wieder mit Beispielen aus dem Sozialbereich behandelt werden. Auch das Kapitel zum Qualitätsmanagement (QM) in der Sozialen Arbeit leugnet keinesfalls den ökonomisch begründeten Ursprung des Themas (S. 133 f.). Über Hinweise auf einschlägige rechtliche Vorschriften, die dem Sozialbereich ein QM in unterschiedlicher Strenge vorgeben, werden bekannte branchenübergreifend konzipierte QM-Systeme, wie die DIN EN ISO 9000ff. und EFQM, verbunden mit Hinweisen auf Besonderheiten sozialer Dienstleistungen behandelt.

Dass in den Texten zur Wirtschaftlichkeit und Finanzierung die betriebswirtschaftliche Perspektive evident und dominant ist, bedarf keiner näheren Begründung. 


\section{b) Gesamtwürdigung im Lichte des Besonderheitsanspruchs der Managementperspektive}

Die von Bieker und Vomberg herausgegebene Publikation hält im Grunde das, was der Titel beim genauem Hinsehen verspricht: Es wird Management behandelt, das sich in Einrichtungen bzw. Tätigkeitsfeldern der Sozialen Arbeit vollzieht. Die Texte hätten mit anderen Beispielen so auch für andere Branchen geschrieben worden sein können. Dem klassischen Standard von Lehrbüchern zur Betriebswirtschaft oder zum Management entsprechen im engeren Sinne sechs, ggf. sieben der zehn Kapitel. Einzig die Beiträge zu „Sozialer Arbeit und Sozialmanagement“ und zum Teil die Beiträge zum Thema „Diversity Management“ heben in erkennbarem Mindestmaß Besonderheiten und Begründungszusammenhänge aus einer explizit Sozialen Perspektive hervor, wenn etwa das Diversity-Thema auf Menschenrechte bezogen wird. Nicht in jedem Fall bleibt dann wiederum aber der Bezug zum Management erhalten. Dass aus dem theoretischen Bereich der Sozialen Arbeit ein Beitrag für eine wie auch immer verstandene integrierte (Sozial-)Managementperspektive geleistet wird, das wird in dem Werk weder behauptet, noch - soweit erkennbar - versucht. Der interessierte Praktiker aus sozialen Organisationen findet somit weitgehend schnörkellos dargestelltes betriebswirtschaftliches Anwendungswissen, übertragen auf soziale Einrichtungen und Dienste.

\section{Böttcher/Merchel: „Einführung in das Bildungs- und Sozialmanagement““}

\section{a) Themenbreite und inhaltliche Analyse}

Böttcher und Merchel befassen sich mit Sozial- und Bildungsmanagement in einem gemeinsamen Lehrbuch. Eine Begründung für diesen doppelten Gegenstandsbereich erfolgt nicht. So beschreiben die Autoren ausführlich gerade die Unterschiedlichkeit der Bereiche (S. 11 f.). Als Gemeinsamkeit wird allenfalls aufgeführt, dass alle Organisationen gleich welcher Branche und Ausrichtung identische Probleme zu lösen haben (S. 25). Insgesamt liegt der Schwerpunkt der Ausführungen eher im Bereich des Sozial- als im Bildungsmanagement. Nach einer Unterscheidung von Regieren und Managen widmet sich der Kern des Buches dem Feld Handlungsbereiche des Managements. Ausgehend von einem inhaltlichen Gesamtumfang (d. h. ohne Editorial Inhaltsverzeichnis, Glossar, Literatur) von 220 Seiten befasst sich der Hauptteil des Buches mit folgenden Handlungsbereichen:

- Organisationsbezogene Steuerung (58 S.)

- Betriebswirtschaftliche Steuerung (32 S.)

- Fachliche Steuerung: Qualitätsmanagement und Evaluation (31 S.)

- Personalmanagement: Rekrutierung und Entwicklung des Personals (28 S.)

- Gestaltung der Beziehung der Organisation zu ihrer Umwelt (9 S.)

- Bildungs- und Sozialmarketing als umfassender Managementansatz (28 S.)

Innerhalb der einzelnen Handlungsfelder werden wiederum ausgewählte Schwerpunkte gelegt. So liegt der Fokus der organisationsbezogenen Steuerung auf den Themen Organisationsentwicklung bzw. Change Management und dem Projektmanagement. Betriebswirtschaftliche Steuerung setzen die Autoren ausschließlich gleich mit Finanzierungsmanagement/Rechnungswesen, 
womit sie auch entsprechende Themen (wie. z. B. Finanzierungsformen, Budgetierung, Fundraising) an dieser Stelle behandeln. Dieses irritiert, zumal sie sich bewusst sind, dass Finanzierungsfragen nur ,ein wichtiger Bestandteil des Managements in Einrichtungen der Sozialen Arbeit [darstellen]“ (S. 98). Im Handlungsfeld Personalmanagement liegt der Fokus auf Teilfunktionen der Rekrutierung, Personalentwicklung, Motivation sowie - unerklärlicher Weise als Exkurs auf sozialen Kompetenzen. Alle anderen Funktionen des Personalmanagements werden nicht bzw. nur kurz (S. 164 f.) angesprochen. Weshalb in einer Branche, die z. T. bis zu 80\% Personalkostenanteil verzeichnet, gerade das Personalmanagement nicht zu einer finanzwirtschaftlich verstandenen betriebswirtschaftlichen Steuerung gezählt wird, hätte einer Erklärung bedurft.

\section{b) Gesamtwürdigung im Lichte des Besonderheitsanspruchs der Managementperspektive}

Die Arbeit von Böttcher und Merchel ist bemüht um eine ideologie- und vorurteilsfreie Adaption betriebswirtschaftlicher und managerialer Methoden auf den Sozialbereich. Sachlich stellen sie eine generelle Hinwendung des sozialen Bereichs zu Managementmethoden fest, freilich nicht ohne den obligatorischen Warnhinweis vor einer unreflektierten Anwendung auszukommen (S. 98 u. 131). Es spricht von einem hohen Maß an Realitätsnähe, wenn sie dafür eintreten, dass „betriebswirtschaftliches Denken als eine legitime Steuerungsform in Einrichtungen des ... Sozialwesens von den dort tätigen Fachkräften akzeptiert wird ..." (S. 98). Im Hinblick auf das Sozialmanagement plädieren die Autoren „für eine integrierte Management-Perspektive“, die ökonomische Denktraditionen mit denen der Sozialen Arbeit verknüpft (S. 131). Worin allerdings konkret das soziale Element in den fünf von sechs Handlungsbereichen des Managements liegt, bleibt genau so unscharf, wie umgekehrt das ökonomische/manageriale Element im Handlungsbereich der sozial-fachlichen Steuerung. Von Argumentationen sozialer/pädagogischer Art sind in den Handlungsfeldern nur Spurenelemente und zwar ausgerechnet als Exkurs erkennbar. So gesehen erscheint der inhaltliche Beitrag der 'Sozialen Disziplinen' zum Sozialmanagement überschaubar. Bezogen auf das gesamte Kapitel Handlungsbereiche des Managements (183 Seiten) entspricht die sozial- (und bildungs-) fachliche Steuerung gerade einem Anteil von rund 17\%, damit entfallen etwa $83 \%$ auf die ökonomisch/managerialen Perspektive. Die betrachteten Bereiche decken dabei in der Breite thematisch alle Fragen ab, wie sie sich auch in Einführungslehrbüchern zum allgemeinen Management oder zur Betriebswirtschaftslehre finden lassen. Einzig das Qualitätsmanagement und die Evaluation fallen als vermeintliche Sonderbereiche des Sozialen und Pädagogischen gegebenenfalls in der vorliegenden Darstellung aus dem Rahmen üblicher Managementlehrbücher.

\section{Schellberg: „Betriebswirtschaftslehre für Sozialunternehmen“}

\section{a) Themenbreite und inhaltliche Analyse}

Klaus Schellberg verfolgt mit seinem in der 5. Auflage erschienenen Lehrbuch das „Kernanliegen ... eine eigene Betriebswirtschaftslehre der Sozialunternehmen zu entwickeln.“ (S. 16). Aus- 
drücklich sieht der Autor seinen Ansatz als spezielle Betriebswirtschaftslehre (BWL) für Sozialunternehmen konzipiert und zeichnet Konturen einer solchen speziellen BWL auf (S. 45 f. u. 46ff.). Dabei grenzt er sich von der verbreiteten Verengung auf Nonprofit Organisationen ab (S. 46), die das Unternehmensspektrum der Sozialwirtschaft nur teilweise erfassen. Die spezielle BWL der Sozialunternehmen habe vielmehr die „Besonderheiten der Dienstleistungsbetriebswirtschaft" aufzunehmen (S. 47). Das Lehrbuch umfasst 14 Kapitel mit folgenden Inhalten:

- Sozialunternehmen als Gegenstand der Ökonomie (20 S.)

- Die Grundlegungen der BWL von Sozialunternehmen (28 S.)

- Das Rechnungswesen (22 S.)

- Controlling (9 S.)

- Die Rechtsformen (6 S.)

- Strategische Unternehmensführung (18 S.)

- Beschaffung (5 S.)

- Dienstleistungsproduktion (17 S.)

- Personalwirtschaft (14 S.)

- Marketing (14 S.)

- Finanzwirtschaft und Finanzmanagement (9 S.)

- Finanzierung (15 S.)

- Sozialwirtschaftliche Finanzierung (33 S.)

- Unternehmensethik sozialer Unternehmen (3 S.)

Die Auswahl der Themen entspricht weitgehend derjenigen, wie sie in allgemeinen betriebswirtschaftlichen Lehrwerken zu finden ist; einzig das Thema Organisation fehlt. Besondere Aufmerksamkeit erfährt das Thema Finanzierung. In drei Kapiteln $(11,12,13)$ werden auf 57 Seiten Finanzierungsfragen erörtert, was nahezu $25 \%$ der inhaltlichen Textseiten umfasst. Berücksichtigt man weitere 31 Seiten für die Themen Rechnungswesen und Controlling, dann umfassen mehr als ein Drittel des Buches klassische, eher quantitative betriebswirtschaftliche Themen.

Die beiden ersten Kapitel (S. 23-71) sind von dem Gedanken getragen, Sozialunternehmen als Gegenstand einer spezielle BWL auch theoretisch in die ökonomische Theorie einzubinden. So wird z. B. das Ökonomische Prinzip auf Sozialunternehmen übertragen und Sozialunternehmen in klassische Kreislaufmodelle eingebunden. Im zweiten Kapitel erfolgt insbesondere die theoretisch-konzeptionelle Begründung für eine spezielle BWL der Sozialunternehmen, indem Besonderheiten der Dienstleistung Soziale Arbeit aufgezeigt werden. Der Autor selbst bemüht sich nicht den Begriff Sozialmanagement mit Ansätzen Sozialer Arbeit zu verbinden. Es entsteht somit keinerlei Unschärfe. Der Terminus Sozialmanagement befindet sich nicht einmal im Stichwortverzeichnis. Gleichwohl ordnet das von Gotthard Schwarz verfasste Vorwort die Arbeit in die „Diskussion um das Sozialmanagement“ (S. 16) ein. Der Autor selbst sieht seine Ausführungen zwar in der Buchreihe zum Sozialmanagement eingebettet, betont aber gleichzeitig, dass seine Themen nur den „harten Kern“ (S. 22) der BWL umfassen. Vermeintlich „weiche Themen“ wie Führung, Organisationsentwicklung etc. sind bewusst ausgeklammert. Entsprechend werden Themen wie z. B. Personal explizit als Personalwirtschaft und nicht als Personalmanagement ausgewiesen. Ausführlich behandelt werden dann quantitative Ansätze etwa zur Personalbedarfsberechnung oder Personalkostenrechnung (159ff.). Den Anspruch einen Beitrag für eine spezielle BWL für Sozialunternehmen leisten zu wollen (S. 45), wird Schellberg in der Weise gerecht, dass 
er zu allen Teilfunktionen - wenn auch z. T. kurz - praxisbezogene Besonderheiten für den Sozialbereich hervorhebt: So wird im Rechnungswesen z. B. auf so genannte Verwendungsnachweise (S. 73) und die Steuerbefreiung von Sozialorganisationen (S. 75 f.) hingewiesen und im Bereich der Rechtsformwahl wird die Sonderstellung der Kirchen thematisiert (S. 107). Das Kapitel zur Strategischen Unternehmensführung weist beispielsweise auf die außergewöhnliche Bedeutung von Stakeholdern (S. 123) hin und beschreibt den ,ethischen Auftrag“ und die „Refinanzierbarkeit“ (S. 128) als zwei Merkmale von Geschäftsfeldern sozialer Dienstleister. Besonderheiten im Personalbereich werden etwa in der Mischung von Professionellen und Laien erkannt (S. 155) und den Grundlagen der Finanzierung von sozialwirtschaftlichen Organisationen wird ein ganzes Kapitel gewidmet.

\section{b) Gesamtwürdigung im Lichte des Besonderheitsanspruchs der Managementperspektive}

Die von Klaus Schellberg vorgelegte „Betriebswirtschaftslehre für Sozialunternehmen“ bietet eine gradlinige Anwendung von klassischer Betriebswirtschaft auf die Besonderheiten von Sozialunternehmen. Ein Lehrbuch, das nicht nur für Studierende und Nicht-Betriebswirte gewinnbringend zu lesen ist; auch für manche Ökonomen dürften die Besonderheiten von Unternehmen des Sozialbereichs eine unbekannte Größe darstellen. Obgleich das Buch in der Tradition des so genannten Sozialmanagements steht, unternimmt Schellberg keinerlei Verrenkungen eine wie auch immer geartete Integration von 'Wirtschaft' und 'Sozialem' zu versuchen. Vielmehr gelingt es ihm verständlich darzulegen, aufgrund welcher konzeptionellen Überlegungen soziale Organisationen Gegenstand einer speziellen BWL sein können.

\section{Wöhrle/Beck/Grunwald/Schellberg/Schwarz/Wendt: „Grundlagen des Managements in der Sozialwirtschaft"“}

\section{a) Themenbreite und inhaltliche Analyse}

Der von sechs Autoren als Gemeinschaftswerk verfasste Band erscheint als Teil der Reihe Studienkurs Management in der Sozialwirtschaft im Nomos Verlag. In sechs inhaltlich nicht zusammenhängenden Kapiteln werden grundsätzliche allgemeine Fragestellungen behandelt, die unmittelbar praxisnahe Probleme überwiegend ausklammern.

- Sozialwirtschaft (Wendt, W. R., 27 S.)

- Sozialstaat, Sozialpolitik und (sozial-) politische Steuerung (Beck, R./Schwarz, G., 43 S.)

- Soziale Arbeit, ihre Selbstversorgung und ihr Verhältnis zu Fragen der Steuerung sozialwirtschaftlicher Unternehmen (Grunwald, K., 35 S.)

- Die Wirtschaftswissenschaften und ihr Verhältnis zur Sozialwirtschaft (und der Sozialen Arbeit) (Schellberg, K., 39 S.)

- Organisationstheorien und Managementlehre (Wöhrle, A., 33 S.)

- Sozialmanagement und Management in der Sozialwirtschaft (Wöhrle, A., 42 S.) 
Das als Lehrbuch zum Management in der Sozialwirtschaft konzipierte Werk unterscheidet sich grundlegend von den oben besprochenen Publikationen. Nahezu alle Kapitel befassen sich zu wesentlichen Teilen mit dem Selbstverständnis des Sozialmanagements bzw. des Managements in der Sozialwirtschaft und seiner Abgrenzung zu der Wirtschaftswissenschaft.

Wendt (1. Kapitel) entfaltet sein Verständnis von Sozialwirtschaft, das diese als Bedarfswirtschaft sieht, die in gesellschaftlichem Kontext die Versorgung leistet (S. 9). Dazu skizziert er mit Hilfe einer historischen Herleitung einen (sozialen) Wirtschaftsbegriff. Dieser basiert im Wesentlichen auf der etymologischen Bedeutung von Haushalt i. S. v. ,'ganzes Haus' (griech. oikos)“ (S. 10). In diesem Urtypus einer Versorgungsgemeinschaft sei Wirtschaften gleichbedeutend mit „Haushalten und meint von vornherein eine sozial bestimmte Aufgabe.“ Im Kontext der gewählten Interpretation der Versorgungsgemeinschaft wird als Kernfunktion der Sozialwirtschaft gesehen, wie die Wohlfahrtspflege ,als nötig erkannte Versorgung tatsächlich erfolgt..." (S. 18). Die Rolle der Sozialwirtschaft wird dabei eingebunden in sozialpolitische Entscheidungen über den sozialen Bedarf, der dann entsprechend zu finanzieren sei. Das Handeln und Wirken der Akteure der Sozialwirtschaft wird vom Autor, mit einer gewissen Distanz zu betrieblichen Praxis- oder Managementfragen, im Wesentlichen von der Makro- und Mesoebene aus beschrieben.

Das 2. Kapitel von Beck und Schwarz befasst sich nahezu ausschließlich mit sozialpolitischen Fragestellungen, indem beispielsweise historische Entwicklungen der Sozialpolitik beschrieben und Konstruktionsprinzipien des deutschen Sozialstaates ausgebreitet werden. Der Beitrag bzw. das Thema erscheint in einem Werk mit dem vorliegenden Titel etwas übergewichtet. Zum Ende hin wird jedoch der Bezug zum Sozialmanagement wieder hergestellt, indem die Autoren beklagen, dass ,einem 'manageriellen' Verständnis von Sozialmanagement Vorschub geleistet wird“ (S. 70), welches sozialpolitische Bezüge nicht berücksichtigt. Wiederum aus sozialpolitischer Perspektive wird die Erwartung formuliert, dass sich die Vertreter des Sozialmanagements an Diskussionen des Sozialstaates aktiv beteiligen sollten (S. 71).

Klaus Grunwald (3. Kap.) bemerkt in seinem Beitrag ein ignorierendes Nebeneinander „Sozialpädagogischen Denkens“ und Sozialmanagement. Der Beitrag beschäftigt sich im Kern mit der Frage nach dem Selbstverständnis der Sozialen Arbeit, die er auch als wissenschaftliche Disziplin begreift. Insbesondere die Perspektiven Soziale Arbeit als Wissenschaft und als Profession werden voneinander abgegrenzt (S. 77ff.). Allgemein werden Steuerungskonzepte (Steuerung, Regelung, Lenkung, Kybernetik) rund um das Thema Management sozialwirtschaftlicher Organisationen darstellt, wie sie auch aus dem Bereich der unternehmensstrategischen Planungslehre bekannt sind (S. 83 f.). Gleiches gilt für die Ablehnung plandeterministischer Steuerung (S. 85). Grunwald fordert Organisations- und damit Managementfragen sozialer Dienstleistungen nicht an andere wissenschaftliche Disziplinen auszulagern, sondern durch die Soziale Arbeit selber aufzugreifen (S. 100). Greifbare Aussagen wie oder mit welchen konkreten Inhalten dieses geschehen könnte, bleiben jedoch mit den Hinweisen auf eine so genannte lebensweltorientierte Soziale Arbeit (S. 86ff.) eher im Grundsätzlichen, jedenfalls sofern es um die Lösung von Problemen mit Praxisbezug geht. Dies ist nur konsequent, da Grunwald vielmehr die Notwendigkeit sieht, das Sozialmanagement ,weg von den Interna sozialer Dienstleistungsunternehmen hin zu einer sozialpolitischen Perspektive“"(S. 101) zu bewegen und den Schwerpunkt auf eine umfassende kommunale, also sozialpolitische, Planung zu legen (S. 102). Es fragt sich allerdings, ob genau dadurch 
nicht interne Organisationsfragen, wie oben noch abgelehnt, zwangsläufig auf andere Disziplinen ausgelagert werden, um diese Lücke auszufüllen.

Der Beitrag von Schellberg (4. Kap.) zeigt, wie schon oben das Lehrbuch des Autors, das Verhältnis der Wirtschaftswissenschaft zur Sozialwirtschaft und Sozialen Arbeit auf. Treffend ist der Hinweis, dass die Sozialwirtschaft für die Wirtschaftswissenschaft bislang weitgehend terra incognita ist (S. 111). Zutreffend daher auch der konstatierte Forschungsbedarf, da die vorhandene Forschung zu Nonprofit Organisationen diesen Teilbereich, der den Nonprofit Sektor klar dominiert, nicht erfasst (S. 124). Die meisten Sozialunternehmen seien danach keine klassischen Nonprofit-Organisationen (S. 138). Es fragt sich allerdings, was vom Nonprofit-Sektor noch übrig bliebe, zählte man die Sozialunternehmen der freien Wohlfahrtspflege nicht (mehr) hinzu. Insgesamt wird skizziert, wie aus der Wirtschaftswissenschaft heraus mit den Besonderheiten der Branche Sozialwirtschaft umgegangen werden kann. Soziale Arbeit bezeichnet er aus Sicht der Wirtschaftswissenschaft zutreffend als „Technologie“, zu der i. e. S. die Ökonomik nichts Hilfreiches beitragen kann, genauso wie umgekehrt Soziale Arbeit keine Beiträge zum Umgang mit „physischen Knappheitsproblemen“ liefert (S. 112 f.). Schellberg skizziert somit eine nachvollziehbare Arbeitsteilung der Disziplinen: „Die Soziale Arbeit liefert die Ziele und Inhalte der Arbeit. Die Ökonomie löst die Ressourcenfrage.“ (S. 117). Soziale Dienste sollen daher also Sozialpolitik verwirklichen (S. 114) und nicht - etwa wie oben bei Grunwald und Beck/Schwarz gefordert - betreiben. Fernab von den sonst in der Literatur vorzufindenden engagierten Verweisen auf die vorgeblichen Besonderheiten der Dienstleistung Soziale Arbeit bzw. von Sozialunternehmen, arbeitet Schellberg betont sachlich ökonomische Besonderheiten von Sozialer Arbeit als Dienstleistung (S. 129 f.) heraus und benennt Konsequenzen für alle betrieblichen Grundfunktionen (Beschaffung, Produktion, Controlling etc.) (131ff.). Die in der Sozialen Arbeit geführte Diskussion einer so genannten Ökonomisierung des Sozialen wird als ein „Scheinproblem“ erkannt, da die Wirtschaftswissenschaft keine, insbesondere keine sozialen Ziele vorgibt, sondern gegebene Ziele ökonomisch-optimal zu erreichen versucht (S. 146).

Armin Wöhrle steuert die beiden Kapitel 5 und 6 zu dem Werk bei. In Kapitel 5 erfolgen ein Überblick und eine Einführung in gängige Organisationstheorien (bspw. Taylor, Weber, Systemtheorie) sowie in das Thema Organisationskultur. Darüber hinaus wird klassisches Grundwissen z. B. zum Managementbegriff, -aufgaben und -rollen geboten (bspw. Koontz, O’Donell, Minzberg). Das 6. Kapitel hingegen steht dann ganz im Zeichen einer begrifflichen und konzeptionellen Selbstfindung des „Sozialmanagements“, welches Wöhrle von einem „Management in der Sozialwirtschaft" abgegrenzt. ${ }^{1}$ Zunächst wird der historische Emanzipationsprozess der „Freien Wohlfahrtspflege“ gegenüber der öffentlichen Verwaltung beschrieben, deren „Verlängerter Arm“ sie nicht länger sein wollte (S. 187ff.). Wöhrle zeigt wichtige Etappen eines zunehmend akademisierten Sozialmanagements anhand von Meilensteinen in Praxis und Schrifttum auf. Zum Selbstverständnis Sozialer Dienstleistungen arbeitet Wöhrle den Dienstleistungscharakter Sozialer Arbeit heraus (S. 193ff.) und stellt erhellend fest, dass dieser eben keine Besonderheit darstellt. Ebenso treffend positioniert er sich auch bzgl. der Berücksichtigung von Managementaspekten. Wie in anderen Branchen auch, etwa im Maschinenbau (S. 217), müsse betriebswirtschaftliche Managementkompetenz berücksichtigt werden. Im Grunde eine Selbstverständlichkeit, die sich

1 Hierzu wird im Text ausdrücklich, weil z.T. deckungsgleich, hingewiesen auf Wöhrle (2013). 
vermutlich an den kritischen sozialwissenschaftlich geprägten Leser richtet. Das eigentliche Anliegen des Beitrags liegt aber in der terminologischen Abgrenzung von Sozialmanagement und Management in der Sozialwirtschaft. Den Begriff Sozialmanagement sieht Wöhrle eng mit der so genannten Fachlichkeit Sozialer Arbeit, also spezifisches Wissen Sozialer Arbeit und ihren Standards verbunden (S. 217). Es geht bei diesem Begriff somit nicht allein um die (wirtschaftliche) Überlebenssicherung des Sozialunternehmens, sondern auch und gerade um z. B. sozialpolitische Zusammenhänge (S. 220). „Management in der Sozialwirtschaft“ hingegen ist für den Autor mehr bzw. etwas anderes als Sozialmanagement. Er sieht den Terminus auch auf alle anderen Tätigkeitsbereiche der Wohlfahrtsverbände/Sozialwirtschaft übertragbar, d. h. auch im Gesundheits-, Pflege-, Bildungs- und Kulturbereich (S. 221). Die Soziale Arbeit i. e. S. tritt hier in den Hintergrund, auch wenn dafür plädiert wird, dass die Soziale Fachlichkeit im Management vorhanden sein sollte (S. 217).

\section{b) Gesamtwürdigung im Lichte des Besonderheitsanspruchs der Managementperspektive}

Das Buch ist nicht als Herausgeberwerk, sondern als Gemeinschaftswerk ausgewiesen. Angesichts des Titels „....Management in der Sozialwirtschaft“ und der von Wöhrle vorgenommenen Begriffsdifferenzierung von Sozialmanagement und Management in der Sozialwirtschaft, erscheinen die Beiträge von Beck und Schwarz sowie der von Grunwald daher erklärungsbedürftig. Insgesamt lassen sich aber alle Beiträge der zentralen Frage zuordnen, welche Rolle die Soziale Arbeit - insbesondere wenn man sie als Wissenschaft verstehen will - im Sozialmanagement spielen kann und sollte. Abstandsbemühungen zur Betriebswirtschaftslehre sind in dem Werk durchgängig vorhanden. Ein eigenständiger fachspezifischer Beitrag, der auch für praxisnahe Fragestellungen lösungsrelevant wäre, ist indessen nicht erkennbar. Allein die Differenzierung von Wöhrle - und analog auch die Sicht Schellbergs - kann diese Problematik zumindest entschärfen, wenn dies auch in der Konsequenz bedeutet, dass „Management in der Sozialwirtschaft“ ganz überwiegend zu einem Thema der Betriebswirtschaftslehre wird. Ein solches Verständnis liefe am Ende wohl auf eine Herauslösung des „Managements in der Sozialwirtschaft“ aus dem unmittelbaren Betrachtungsbereich der Sozialen Arbeit hinaus und würde den oben formulierten Besonderheitsanspruch hinfällig machen.

\section{Fazit: „Was ist Sozialmanagement?“}

Die Untersuchung der vorgenannten Lehrwerke verdeutlicht, dass das in Abschnitt 1 skizzierte Akzeptanzproblem bzgl. der Anwendung von Managementkonzepten in der Sozialen Arbeit keinesfalls unbegründet ist.

Von besonderen, im Sinne von neuen Managementkonzepten, die so nicht auch schon im Kern ein etablierter Bestandteil der (betriebswirtschaftlichen) Managementlehre sind, ist insgesamt allenfalls ein Silberstreif am Horizont erkennbar. Die Beiträge der Disziplin der Sozialen Arbeit zum Sozialmanagement spielen sich eher auf einem makro- bzw. sozialpolitischen Plateau ab. Sie bleiben vage und auf der Ebene von Forderungen. Für konkrete Managementfragen wird über- 
wiegend auf Darstellungen zurückgegriffen, die - abgesehen von den Beispielen - so auch tendenziell in einfach gehaltenen Einführungsbüchern zur Allgemeinen Betriebswirtschaftslehre zu finden sein könnten. Hilfreich und klärend ist die Differenzierung von Sozialmanagement und Management in der Sozialwirtschaft, wobei letzterer Terminus konsequent zu Ende gedacht auf eine spezielle BWL des Sozialbereichs, ähnlich einer Bank- oder Handelsbetriebslehre etc. hinausläuft. Es entsteht leicht der Eindruck, als habe sich das Thema (nur) deshalb an den (Fachhochschul-)Fakultäten für Soziale Arbeit bzw. Sozialwissenschaften etabliert, weil betriebswirtschaftliche Fakultäten sich dieses Themas bislang nicht angenommen haben. Es über die Nonprofit-Sektor Forschung mit erfassen zu wollen, greift zu kurz.

Sozialmanagement führt somit derzeit ein ambivalentes Dasein. Obgleich es, insbesondere in der Interpretation als „Management in der Sozialwirtschaft“, aufgrund der vermittelten Inhalte im Grunde Merkmale einer sich entwickelnden speziellen BWL der Sozialwirtschaft aufweist, erfolgt die entsprechende Lehre und Forschung nahezu vollständig an Fachbereichen für Soziale Arbeit bzw. angewandter Sozialwissenschaften und dort wiederum oftmals von Ökonomen. Eine für Wissenschaft und Praxis gleichermaßen unbefriedigende Konstellation. Um Sozialmanagement tatsächlich zu einem integrierten Managementsatz des Sozialbereichs zu entwickeln, bedarf es vor allem spezifischen praxisrelevanten Inputs aus dem Bereich der nicht-betriebswirtschaftlichen Sozialwissenschaften, der über abstrakte Darstellungen und der Formulierung (sozial-) politischer Forderungen erkennbar hinausgeht.

\section{Literaturverzeichnis}

Bei der mit * gekennzeichneten Literatur handelt es sich um die im Text besprochenen Werke.

Albert, M. (2006): Die Ökonomisierung der Sozialen Arbeit, in: Sozial Extra, Heft 7/8 2006, S. 26-31.

Bieker, R./Vomberg, E. (Hrsg.) (2012)*: Management in der Sozialen Arbeit, Stuttgart

Böttcher, W./Merchel, J. (2010)*: Einführung in das Bildungs- und Sozialmanagement, Opladen \& Farmington Hills, MI

Brinkmann, V. (2010): Sozialwirtschaft, Wiesbaden

Flösser, G./Otto, H.-U. (Hrsg.) (1992): Sozialmanagement oder Management des Sozialen?, Bielefeld

Gehrmann, G. (1993): Management in sozialen Organisationen, Berlin/Bonn/Regensburg

Grams, W. (2000): Soziale Arbeit als Ware oder: Das Soziale zu Markte tragen, in: Wilken, U. (Hrsg.): Ethik und Ökonomie, Freiburg im Breisgau, S. 77-98.

Maelicke, B. (2008): Lexikon der Sozialwirtschaft, Baden-Baden

Müller-Schöll, A./Priebke, M. (1989): Sozialmanagement: Zur Förderung systematischen Entscheidens, Planens, Organisieren, Führens und Kontrollierens in Gruppen, Neuwied/Kriftel/Berlin

Prokla (2007): Ausgabe 148: Verbetriebswirtschaftlichung, Zeitschrift für kritische Sozialwissenschaft, 37. Jg. Nr. 3 Sep. 2007

Schellberg, K. (2012)*: Betriebswirtschaftslehre für Sozialunternehmen, 5. Aufl., Ziel Verlag, Augsburg

Schwarz, G. (1994): Sozialmanagement, München

Wöhrle, A. (2013): Mit welchen Begriffen des Managements argumentieren wir?, in: Kölner Journal. Wissenschaftliches Forum für Sozialwirtschaft und Sozialmanagement, Heft 1/2013, S. 34-59.

Wöhrle, A./Beck, R./Grunwald, K./Schwarz, G./Wendt, W.R. (2013)*: Grundlagen des Managements in der Sozialwirtschaft, Baden-Baden

Zimmer, A./Nährlich, S./Friedrich, P. (2009): Zur volkswirtschaftlichen Bedeutung der Sozialwirtschaft, in: Lehrbuch der Sozialwirtschaft, 3. Aufl., hrsg. von Arnold, Ulli/Maelicke, Bernd, Baden-Baden, S. 117-133. 